\title{
Comparison of outcomes for supine vs. prone position ERCP: a systematic review and meta-analysis
}

\section{(ㄷ)(잉}

\section{Authors}

Harmeet Singh Mashiana ${ }^{1,{ }^{*},}$ Mahendran Jayaraj1,2, ", Babu Pappu Mohan ${ }^{3}$, Gordon Ohning ${ }^{2}$, Douglas G. Adler ${ }^{4}$

Institutions

1 Department of Internal Medicine, University of Nevada Las Vegas School of Medicine, Las Vegas, NV, USA

2 Division of Gastroenterology, University of Nevada Las Vegas School of Medicine, Las Vegas, NV, USA

3 Department of Internal Medicine, University of Alabama, Tuscaloosa, AL, USA

4 Gastroenterology and Hepatology, University of Utah School of Medicine, Huntsman Cancer Center, Salt Lake City, UT, USA

submitted 6.11.2017

accepted after revision $\quad$ 12.3.2018

\author{
Bibliography \\ DOI https://doi.org/10.1055/a-0603-3302 | \\ Endoscopy International Open 2018; 06: E1296-E1301 \\ (c) Georg Thieme Verlag KG Stuttgart · New York \\ ISSN 2364-3722
}

Corresponding author

Douglas G. Adler, MD, Gastroenterology and Hepatology, University of Utah School of Medicine, Huntsman Cancer Center, 30 N 1900 E, Room 4R118, Salt Lake City, Utah 84132, USA

Fax: +1-801-581-8007

douglas.adler@hsc.utah.edu

\section{ABSTRACT}

Background While endoscopic retrograde cholangiopancreatography (ERCP) is usually performed in the prone position, some studies have advocated for ERCP in the supine position. Studies comparing the technical success and safety outcomes have shown variable results. We performed a systematic review and meta-analysis of studies reporting the comparison between the two positions for ERCP outcomes.

Methods We conducted a search of electronic databases and conference proceedings including PubMed, EMBASE, and Web of Science databases (from inception through October 2016) to identify studies that reported the comparison of technical success and safety outcomes between supine and prone ERCP. The primary outcome was to estimate the pooled rates of technical success. The secondary outcome was to estimate the risks of complications, such as cardiopulmonary and post-ERCP pancreatitis (PEP).

Results Six studies reporting on 309 supine and 1415 prone ERCPs were identified. The pooled technical success rates for completion of ERCP in supine and prone positions were $89.1 \%(95 \% \mathrm{Cl}=80.9-94.0)$ and $95.6 \%(95 \% \mathrm{Cl}=$ $91.5-97.7)$, respectively. The pooled rates for complications (cardiopulmonary and PEP) in the supine position were $37.5 \%(95 \% \mathrm{Cl}=19.1-60.3)$ and $3.5 \%(95 \% \mathrm{Cl}=1.6-$ 7.3 ), respectively. The pooled rates for complications (cardiopulmonary and PEP) in the prone position were $41.0 \%$ $(95 \% \mathrm{Cl}=20.9-64.8)$ and $3.9 \%(95 \% \mathrm{Cl}=2.4-6.4)$, respectively. The mean time required for the procedure was 30 minutes and 29.8 minutes for supine and prone positions, respectively. Substantial heterogeneity was noted in the analysis.

Conclusion Prone ERCPs have a higher technical success rate with a slightly lower mean duration but a higher number of adverse events. The decision with regard to patient position should be made after evaluating the overall clinical scenario.

\section{Introduction}

Endoscopic retrograde cholangiopancreatography (ERCP) is an important diagnostic and therapeutic modality in diagnosing and treating innumerable pancreatobiliary pathologies [1]. It is a technically difficult procedure with the potential risk of sever-

\footnotetext{
* These authors contributed equally.
}

al serious complications including cholangitis, pancreatitis, bleeding, and anesthesia-related cardiopulmonary complications [2].

ERCP is most commonly performed with the patient in the prone position or a modified left lateral position [3]. These positions are preferred based upon the consideration that there is lesser aspiration risk, and intubation of the esophagus, stomach, and duodenum is relatively easy. In addition, in this posi- 
tion, secretions do not pool over the ampulla. Furthermore, in the prone position, the endoscopist can face the patient directly.

Some physicians perform ERCP in the supine position. The supine position has an advantage of being a technically easier position for the anesthesiologist, especially in patients who are morbidly obese or who have abdominal wounds and drains [4$6]$. In the supine position, the endoscopist often has to face 180 degrees away from the patient as the rotation of the patient is reflected by a rotation in the endoscope, and this translates into the direction the endoscopist faces.

There is no clear consensus on which position (prone or supine) is superior for performing ERCP.

The present study is a systematic review and meta-analysis of studies reporting the comparison of technical success and safety outcomes of the two ERCP positions.

\section{Methods}

\section{Search strategy}

We conducted a comprehensive search of several databases and conference proceedings including PubMed, EMBASE, and Web of Science databases (earliest inception to October 2016). The search was performed in accordance with PRISMA guidelines to identify studies. The bibliographic section of the selected articles, as well as the systematic and narrative articles on the topic were manually searched for additional relevant articles. Using the NCBI website, a MeSH search restricted to the major MeSH topics was performed using the following topics: (1) endoscopic retrograde cholangiopancreatography; (2) prone position; (3) supine position. These topics were added to the search builder and search commenced in PubMed limited to studies involving humans and published in the English language. Then similar and related articles suggested by PubMed were searched. Articles citing the searched articles were also studied.

\section{Study selection}

Studies that reported on ERCP outcomes based on patient position were included. Both prospective and retrospective studies, and manuscripts and abstracts were included. Only articles in the English literature were considered. The studies were selected by both the first and second authors.

\section{Outcomes assessed}

The primary analysis focused on assessing the outcomes of the ERCP procedure based on patient position. The overall technical success and completion of the ERCP procedure, and complications including cardiovascular complications and post-ERCP pancreatitis (PEP) were measured and analyzed.

\section{Statistical analysis}

We followed the Preferred Reporting Items for Systematic Reviews and Meta-Analyses (PRISMA) guidelines, using a predefined protocol [7]. We used meta-analysis techniques to calculate the pooled estimates in each effect measured, following the methods suggested by DerSimonian and Laird, and our ap- plication can be seen to fit within their general approach (where effect is measured by probability of risk) [8]. The $95 \%$ prediction interval calculated for the studies assessed in our meta-analysis, which deals with dispersion of the effects, varied from -0.56 to 2.42 .

We assessed the heterogeneity between study-specific estimates using two methods. First, Cochran's $Q$ statistical test for heterogeneity was performed, which tests the null hypothesis that all studies in a meta-analysis have the same underlying magnitude of effect [9]. $Q$ is the sum of squared deviations on a standardized scale, and the expected value of $Q$ is equal to the degrees of freedom (number of studies minus 1 ) based on the null hypothesis. When the expected value of $Q$ exceeds the degrees of freedom, the null hypothesis is rejected and variation in effects across studies is accepted to exist, indicating that there is heterogeneity. By convention, the criterion alpha for this test is 0.10 rather than 0.05 since the test typically has low power.

Second, when heterogeneity was present, in order to estimate what proportion of total variances across studies was due to heterogeneity rather than chance, the $R$ statistic was calculated [10]. In this, values of $<30 \%, 30-60 \%, 61-75 \%$, and $>75 \%$ were indicative of low, moderate, substantial, and considerable heterogeneity, respectively. Once heterogeneity was noted, between-study sources of heterogeneity were investigated using sub-group analysis by stratifying original estimates according to study characteristics as described earlier. A $P$ value $<0.1$ for differences between subgroups was considered to be statistically significant. Publication bias was ascertained, qualitatively, by visual inspection of funnel plots [11].

All analyses were performed using Comprehensive MetaAnalysis (CMA) software, version 3 (Biostat, Englewood, New Jersey, USA).

\section{Results}

Six studies reporting on 309 supine and 1415 prone ERCP procedures were identified and a summary of the studies is reported in $>$ Table 1. The pooled technical success rates for completion of ERCP in supine and prone positions, as described in $>$ Table 2 , were $89.1 \%(95 \% \mathrm{Cl}=80.9-94.0)$ and $95.6 \%(95 \% \mathrm{Cl}=$ $91.5-97.7)$, respectively. The pooled rates for complications (cardiopulmonary and PEP) in the supine position were $37.5 \%$ $(95 \% \mathrm{Cl}=19.1-60.3)$ and $3.5 \%(95 \% \mathrm{Cl}=1.6-7.3)$, respectively. The pooled rates for complications (cardiopulmonary and PEP) in the prone position were $41.0 \%(95 \% \mathrm{Cl}=20.9-64.8)$ and $3.9 \%(95 \% \mathrm{Cl}=2.4-6.4)$, respectively. The mean times required for completion of the procedure were 30 minutes and 29.8 minutes for supine and prone positions, respectively. Adverse events secondary to ERCP in these patients are reported in - Table 3.

In the statistical analysis, substantial heterogeneity was noted. The $Q$ value was 39.7, which is substantially higher than the degrees of freedom of 11 . The $R^{2}$ statistic revealed a value of 72.3, which indicates that the proportion of variance across studies due to heterogeneity, rather than chance, was $72.3 \%$. As we used a random effects model in our meta-analysis, the 
- Table 1 Description of studies.

\begin{tabular}{|l|l|l|l|l|l|l|}
\hline Study & Year & Type of study & $\begin{array}{l}\text { Abstract vs. } \\
\text { manuscript }\end{array}$ & $\begin{array}{l}\text { No. of } \\
\text { patients }\end{array}$ & $\begin{array}{l}\text { Males } \\
\text { ERCP }\end{array}$ \\
\hline Trecero et al. [16] & 2010 & Prospective & Abstract & 63 & NR & 28 \\
\hline Nijhawan et al. [15] & 2010 & Prospective cohort & Abstract & 40 & NR & 20 \\
\hline Ferreira and Baron [12] & 2008 & Retrospective & Manuscript & 649 & 340 & 143 \\
\hline Tringali et al. [13] & 2008 & Prospective cohort & Manuscript & 120 & 54 & 60 \\
\hline Diehl [14] & 2006 & Prospective cohort & Abstract & 784 & NR & 41 \\
\hline Terruzzi et al. [4] & 2005 & Prospective cohort & Manuscript & 34 & 21 & 17 \\
\hline
\end{tabular}

- Table 2 Technical success of procedure and duration of procedure in individual studies.

\begin{tabular}{|l|c|c|c|c|c|c|}
\hline Study & $\begin{array}{l}\text { No. of su- } \\
\text { pine ERCP }\end{array}$ & $\begin{array}{l}\text { Success of supine } \\
\text { procedure }\end{array}$ & $\begin{array}{l}\text { No. of prone } \\
\text { ERCP }\end{array}$ & $\begin{array}{l}\text { Success of prone } \\
\text { procedure }\end{array}$ & $\begin{array}{l}\text { Procedure time, } \\
\text { supine }\end{array}$ \\
\hline Diehl [14] & 41 & 36 & 779 & 756 & NR \\
prone
\end{tabular}

- Table 3 Adverse events in individual studies.

\begin{tabular}{|c|c|c|c|c|c|c|}
\hline Study & $\begin{array}{l}\text { No. of supine } \\
\text { procedures }\end{array}$ & $\begin{array}{l}\text { Cardio respiratory } \\
\text { complications, supine }\end{array}$ & $\begin{array}{l}\text { PEP,- } \\
\text { supine }\end{array}$ & $\begin{array}{l}\text { No. of prone } \\
\text { procedures }\end{array}$ & $\begin{array}{l}\text { Cardio respiratory } \\
\text { complications, prone }\end{array}$ & $\begin{array}{l}\text { PEP, } \\
\text { prone }\end{array}$ \\
\hline Diehl [14] & 41 & NR & NR & 779 & NR & NR \\
\hline Nijhawan et al. [15] & 20 & None & 2 & 20 & none & 2 \\
\hline Tringali et al. [13] & 60 & 14 & 1 & 60 & 15 & 1 \\
\hline Terruzzi et al. [4] & 17 & 7 & 1 & 17 & 1 & 1 \\
\hline Ferreira and Baron [12] & 143 & 60 & 2 & 506 & 356 & 16 \\
\hline Trecero et al. [16] & 28 & 13 & 1 & 33 & 18 & 2 \\
\hline Total & 309 & & & 1415 & & \\
\hline
\end{tabular}

$95 \%$ prediction interval, which deals with dispersion of the effects, varied from -0.056 to 2.42 .

- Fig. 1 presents a funnel plot for the studies. Our analysis treated each supine and each prone data as individual entities and hence the 12 data points on the funnel plot for a total of six studies. Based on the funnel plot, we observe that these data points are not symmetrical about the mean, and hence there is a possibility of publication bias. Statistically, we can say that this is more likely due to differences in study and effect size, and inconsistencies of the same across the studies. We can also infer from the plot that there is a possibility of bias based on the position itself.
Overall, we know that the literature is limited in the assessment of ERCP success for the supine vs. prone position, and a publication bias, if any, is going to be negligible; however, statistically, the possibility of a publication bias cannot be ruled out.

Fig. 2 presents a forest plot of technical success rate in the studies.

\section{Discussion}

As described previously in the Results section, the technical success rates are higher in the prone position at $95.6 \%$ with a tighter confidence interval compared to $89.1 \%$ in the supine 
Funnel plot of standard error by logit event rate

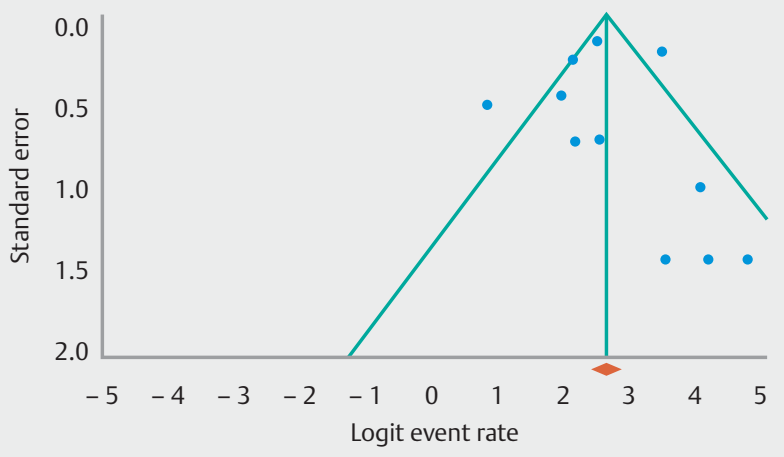

- Fig. 1 Funnel plot graph showing publication bias.

position with a much wider confidence interval. This difference of almost $7 \%$ translates into a clinically significant difference. Our subgroup analysis also showed similar rates of cardiopulmonary and post-ERCP pancreatitis (PEP) complications and very similar mean times required for the completion of procedures in both the supine and prone positions. Hence, our data favor the prone position for a higher technical success rate.

There have been various studies done in the past to evaluate the ERCP outcomes in terms of success rate and complications with patient position as the determinant. Most of the studies have shown very similar technical success rates in the supine and prone positions $[12,13]$. Notably, the study by Terruzzi et al. did show a lower successful cannulation rate of $70 \%$ in ERCP in patients in the supine position compared to $100 \%$ seen in patients undergoing ERCP in the prone position which almost reached a statistically significant difference with a $P$ value of 0.52 [4]. Success rates in the supine position were also significantly lower in the study by Terruzzi et al. than those described in our meta-analysis (70\% vs. $89.1 \%$ ). This can possibly be explained by the limited experience of endoscopists performing procedures in the supine position. Another study by Diehl showed a statistically significantly higher technical success rate in the prone position (95.6\%) compared to the supine position (88\%) [14].

A prospective study by Tringali et al. with 120 patients had an overall success rate of $99.2 \%$ with just one patient in the supine group failing the first-time cannulation due to neoplastic invasion of the duodenal wall [13]. The endoscopists in that study also routinely performed more than $10 \%$ of their procedures in the supine position compared to Terruzzi et al. with less than $5 \%$ supine position procedures $[4,13]$. Another study by Ferreira and Baron with the largest group of ERCPs in the supine position (143) again demonstrated a very similar technical success rate in supine $(90.2 \%)$ and prone $(92.5 \%)$ positions [12]. There was a small difference in success rate favoring the prone position even in that study with a larger supine group, and indeed, there was an overall trend in the data of the studies mentioned favoring the prone position with regard to technical success.

The mean duration of procedures in our meta-analysis was 30 minutes in the supine position and 29.8 minutes in the prone position. The mean duration of procedures was only slightly lower in the prone position. The study by Ferreira and Baron showed a higher mean procedure time of 43 minutes in the supine position vs. 36 minutes in the prone position but the difference was not statistically significant [12]. Their data also favor the prone position since the mean time spent was

\begin{tabular}{|c|c|c|c|c|c|c|c|c|}
\hline \multirow{2}{*}{$\begin{array}{l}\text { Group by subgroup } \\
\text { within study }\end{array}$} & \multirow{2}{*}{$\begin{array}{l}\text { Study } \\
\text { name }\end{array}$} & \multirow{2}{*}{$\begin{array}{l}\text { Subgroup } \\
\text { within study }\end{array}$} & \multicolumn{5}{|c|}{ Statistics for each study } & Event rate and $95 \% \mathrm{Cl}$ \\
\hline & & & Event rate & Lower limit & Upper limit & Z-value & p-Value & \\
\hline prone & Diehl & prone & 0.970 & 0.956 & 0.980 & 15.501 & 0.000 & I \\
\hline prone & Nijhawan & prone & 0.900 & 0.676 & 0.975 & 2.948 & 0.003 & $\rightarrow$ \\
\hline prone & Tringali & prone & 0.992 & 0.882 & 0.999 & 3.377 & 0.001 & 1 \\
\hline prone & Terruzi & prone & 0.972 & 0.678 & 0.998 & 2.479 & 0.013 & $\longrightarrow$ \\
\hline prone & Ferreira & prone & 0.925 & 0.898 & 0.945 & 14.886 & 0.000 & - \\
\hline prone & Trecero & prone & 0.985 & 0.804 & 0.999 & 2.951 & 0.003 & - \\
\hline prone & & & 0.956 & 0.915 & 0.977 & 8.593 & 0.000 & \\
\hline supine & Diehl & supine & 0.878 & 0.739 & 0.948 & 4.136 & 0.000 & $\rightarrow$ \\
\hline supine & Nijhawan & supine & 0.900 & 0.676 & 0.975 & 2.948 & 0.003 & $\longrightarrow$ \\
\hline supine & Tringali & supine & 0.983 & 0.891 & 0.998 & 4.043 & 0.000 & - \\
\hline supine & Terruzi & supine & 0.706 & 0.458 & 0.872 & 1.645 & 0.100 & $\longrightarrow$ \\
\hline supine & Ferreira & supine & 0.895 & 0.833 & 0.936 & 7.856 & 0.000 & - \\
\hline supine & Trecero & supine & 0.929 & 0.755 & 0.982 & 3.495 & 0.000 & $\rightarrow$ \\
\hline supine & & & 0.891 & 0.809 & 0.940 & 6.264 & 0.000 & $\bullet$ \\
\hline \multirow[t]{2}{*}{ Overall } & & & 0.928 & 0.888 & 0.954 & 10.448 & 0.000 & $\sqrt{1}$ \\
\hline & & & & & & & & 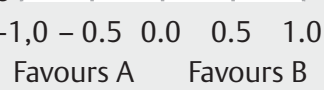 \\
\hline
\end{tabular}

Fig. 2 Forest plot of technical success rate in the studies. 
higher for the supine position even though the technical success rate was slightly lower. The study by Terruzzi et al. showed similar time differences favoring the prone position (33.4 minutes vs. 29.4 minutes) which were close to the outcomes of our data. The mean duration of procedures was 23 minutes for both supine and prone positions in the study by Tringali et al. [13]. The procedure times described by Nijhawan et al. were $13 \pm 4$ minutes in the supine position and $15 \pm 5$ minutes in the prone position which were lower than the rates in our meta-analysis but there was no detailed procedural description to ascertain the reason for the lower procedure times [15]. Trecero et al. also described a lower procedure time in supine patients (38 minutes) than in prone patients (46 minutes) but the difference did not reach statistical significance [16].

Adverse events were divided into cardiopulmonary complications and post-ERCP pancreatitis (PEP). In our analysis, the rates of cardiopulmonary complications were $37.5 \%$ in supine and $41 \%$ in prone positions. A difference of $3.5 \%$ might prove to be a clinically significant difference looking at the ever-increasing number of ERCPs that are currently being performed. In the study by Ferreira and Baron, the complication rates were $75 \%$ in the supine position and $70 \%$ in the prone position with no statistically significant difference between them [12]. They had a lower threshold for describing the cardiopulmonary complications, which can potentially explain the higher rates of complications. Terruzzi et al. described the cardiopulmonary complications to be statistically significantly higher in patients in the supine position at $41 \%$ vs. patients in the prone position at $6 \%$ [4]. The threshold used to describe a cardiopulmonary complication was high, hence, the lower overall rates of complications compared to Ferreira and Baron [12]. These two studies are outliers when compared to the other studies.

The other adverse event included in our meta-analysis, PEP, had similar complication rates in the supine $(3.5 \%)$ and prone positions (3.9\%). Even though the actual rate of PEP is lower by $0.4 \%$ in the supine position it has a wider $95 \% \mathrm{Cl}$ of $1.6-$ $7.3 \%$ compared to $2.4-6.4 \%$ in the prone position. This demonstrates that the concern for PEP should not be a limiting factor for choosing one position over the other.

The prone and left lateral decubitus positions have traditionally been the preferred positions for performing ERCP based upon the consideration that there is a lower aspiration risk and relatively easy intubation of the esophagus $[4,5,15]$. For intubated patients under general anesthesia, some anesthesiologists prefer the supine position because of ease of monitoring [4]. It is also a comfortable position for the patient. Continuous oral suction in the supine position can help to reduce the risk of aspiration [12]. The supine position is also favored by the patient and anesthesiologist in certain conditions when the patients are morbidly obese, have abdominal wounds, drains, percutaneous gastrostomy or jejunostomy tubes. Patients with altered anatomy such as hilar biliary strictures, previous Billroth II gastrectomy or pancreatic duct anatomy which is already known to be difficult to interpret are also preferred for supine position ERCP $[4,5,13]$.

Some of the practical hindrances for performing supine position ERCP have been the orientation of the camera in the scopes requiring the endoscopist to face away from the patient. The pooling of secretions in the duodenum in the supine position hinders visualization of the papilla making the procedure more difficult. For obvious reasons, the supine position is comfortable for the patient and anesthesiologist whereas the prone position is comfortable for the endoscopist thereby increasing the technical success and shortening the duration of the procedure. This fact is proven by the data from our meta-analysis. Some of the reasons for better technical success in the prone position can be explained by the fact that most endoscopists learn to perform ERCP in the prone position and are more comfortable performing ERCPs in this position thereby facilitating effective cannulation of the papilla and obtaining quality radiographs [5].

We acknowledge the limitations in the current meta-analysis. First, substantial heterogeneity was noted in the analysis due to the different sizes of the studies included. Second, there were only six studies that could be included in our meta-analysis.

In conclusion, the results of our meta-analysis highlight the fact that prone position ERCP is favorable with a slightly higher technical success rate, slightly lower mean duration of the procedure, but a higher adverse event rate. The decision with regard to the position of the patient should be made after carefully evaluating the overall clinical scenario, patient comfort, the endoscopist's experience, and the slightly higher technical success rate for prone position ERCP.

\section{Competing interests}

None

References

[1] Williams E], Taylor S, Fairclough P et al. Are we meeting the standards set for endoscopy? Results of a large-scale prospective survey of endoscopic retrograde cholangio-pancreatograph practice Gut 2007; 56: $821-829$

[2] Olsson G, Arnelo U, Swahn F et al. The H.O.U.S.E. classification: a novel endoscopic retrograde cholangiopancreatography (ERCP) complexity grading scale. BMC Gastroenterol 2017; 17: 38

[3] Yakshe P, Vennes J. Technique of endoscopic retrograde cholangiopancreatography Gastroenterologic Endoscopy.2nd edn. Philadelphia, PA: WB Saunders Company; 2000: 845-862

[4] Terruzzi V, Radaelli F, Meucci $G$ et al. Is the supine position as safe and effective as the prone position for endoscopic retrograde cholangiopancreatography? A prospective randomized study Endoscopy 2005; 37: $1211-1214$

[5] Wilcox CM. Should patients undergoing ERCP be placed in the prone or supine position? Nat Clin Pract Gastroenterol Hepatol 2008; 5: $488-489$

[6] Froehlich F. Patient position during ERCP: prone versus supine. What about left lateral throughout? Endoscopy 2006; 38: 755; author reply 755

[7] Moher D, Liberati A, Tetzlaff ] et al. Preferred reporting items for systematic reviews and meta-analyses: the PRISMA statement. Int J Surg 2010; 8: $336-341$ 
[8] DerSimonian R, Laird N. Meta-analysis in clinical trials. Control Clin Trials 1986; 7: $177-188$

[9] Higgins JP, Thompson SG. Quantifying heterogeneity in a meta-analysis. Stat Med 2002; 21: 1539-1558

[10] Higgins JP, Thompson SG, Deeks JJ et al. Measuring inconsistency in meta-analyses. BMJ 2003; 327: 557 - 560

[11] Sterne JA, Gavaghan D, Egger M. Publication and related bias in metaanalysis: power of statistical tests and prevalence in the literature. J Clin Epidemiol 2000; 53: 1119-1129

[12] Ferreira LE, Baron TH. Comparison of safety and efficacy of ERCP performed with the patient in supine and prone positions. Gastrointest Endosc 2008; 67: $1037-1043$
[13] Tringali A, Mutignani M, Milano A et al. No difference between supine and prone position for ERCP in conscious sedated patients: a prospective randomized study. Endoscopy 2008; 40: 93-97

[14] Diehl D. Supine patient positioning for ERCP: indications and outcomes. Gastrointest Endosc 2006; 63: AB291

[15] Nijhawan S, Jha A, Sharma A et al. ERCP: is it better in supine position under general anaesthesia? Indian J Gastroenterol 2010; 29: A116

[16] Trecero SRP, Acuesta WCR, Purwanta RS et al. A prospective, randomized, open-label comparison of safety, efficacy and success rate of ERCP on prone and supine position. J Gastroenterol Hepatol 2010; 25: A53 